\title{
Design and evaluation of an external filter technique for exposure equalization in mammography
}

\author{
Shyam P. Keshavmurthy, Mitchell M. Goodsitt, Heang-Ping Chan, ${ }^{a)}$ Mark A. Helvie, \\ and Emmanuel Christodoulou \\ Department of Radiology, University of Michigan, 1500 E. Medical Center Drive, Ann Arbor, \\ Michigan 48109-0030
}

(Received 8 July 1998; accepted for publication 16 April 1999)

\begin{abstract}
We are developing an external filter method for equalizing x-ray exposure in the peripheral region of the breast. This method requires the use of only a limited number of custom-built filters for different breast shapes in a given view. This paper describes the design methodology for these external filters. The filter effectiveness was evaluated through a simulation study on 171 mediolateral and 196 craniocaudal view digitized mammograms and through imaging of a breast phantom. The degree of match between the simulated filter and the individual 3-D exposure profiles at the breast periphery was quantified. An analysis was performed to investigate the effect of filter misalignment. The simulation study indicates that the filter is effective in equalizing exposures for more than $80 \%$ of the breast images in our database. The tolerance in filter misalignment was estimated to be about $\pm 2 \mathrm{~mm}$ for the CC view and $\pm 1 \mathrm{~mm}$ for the MLO view at the image plane. Some misalignment artifacts were demonstrated with simulated filtered mammograms. (c) 1999 American Association of Physicists in Medicine. [S0094-2405(99)01108-6]
\end{abstract}

Key words: mammography, equalization, external filtration, simulation

\section{INTRODUCTION}

Radiographic contrast plays a crucial role in the detection of subtle lesions that are signs of early breast cancer. Since radiographic contrast decreases with increasing hardness of the x-ray spectrum, ${ }^{1}$ low-energy radiation and high-contrast screen/film systems are recommended to maximize the contrast between a lesion and the background breast tissue. The low energy x rays used in mammography and reduced tissue thickness at the breast periphery result in a large exposure range in a breast image. We have estimated that the exposure range for a $5-\mathrm{cm}$-thick breast of dense fibroglandular tissue can be as great as $145: 1$ for a $28 \mathrm{kVp} \mathrm{Mo} / \mathrm{Mo}$ spectrum. Since a typical high-contrast film used in mammography provides a narrow latitude in the range of about 10:1, it generally cannot accommodate the wide exposure range of a breast image. ${ }^{2,3}$ The radiographic contrast of mammographic features imaged at the toe and the shoulder regions of the sensitometric curve is greatly reduced. Stacey-Clear et al. ${ }^{4}$ have shown, in their study of breast cancer location in women aged under 50 , that $73 \%$ of the cancers (63 out of 86) were at the periphery of the breast parenchyma, with the majority near the subcutaneous fat. Since the contrast sensitivity of the human visual system also decreases rapidly with an increase in the film density, ${ }^{5-7}$ the poor image quality in the peripheral region imposes a serious limitation on the sensitivity of cancer detection in breasts with dense fibroglandular tissue. These problems may be reduced if an exposure equalization technique can be developed for mammographic imaging.

Several exposure equalization methods have been proposed to improve mammographic imaging. In one method, a water bag $^{8-10}$ or a solid, elastic, unit density x-ray attenuator ${ }^{11}$ fills the gap between the breast and the compression paddle to reduce the breast thickness variation in the peripheral region. This method can be patient-specific. However, it may be difficult to implement, especially for oblique views, when using a water bag. Another method involves scanning of the breast with either single or multiple intensity-modulated x-ray beams that are based upon x-ray transmission signals obtained from single or multiple detectors. ${ }^{12-14}$ Such a method can equalize the exposure throughout the breast rather than just at the periphery. However, the method is complex and requires much greater heat loading of the $\mathrm{x}$-ray tube than conventional mammography and much longer exposure time. The latter may result in significant motion artifacts. Better visualization of lesions at the breast periphery can be achieved by display equalization methods such as hot lighting and postprocessing of digital images. ${ }^{15}$ These methods reduce the contrast threshold for perception. However, they do not improve the signal-tonoise ratio of the image.

We have proposed a new exposure equalization method for reducing the dynamic range of the mammograms. ${ }^{16}$ This method employs a set of external x-ray beam intensity shaping filters that are positioned near the collimator of a mammography system. A similar technique was reported by Boone et al. for equalizing chest radiographs. ${ }^{17}$ However, no $\mathrm{x}$-ray pre-exposure will be required for filter selection in our approach. The proposed mammography equalization system is shown schematically in Fig. 1. A video camera will acquire a color image of a compressed breast. Next, an image segmentation program will be employed to extract the breast boundary. This breast border will then be classified into a breast shape group. A prefabricated exposure equalization 


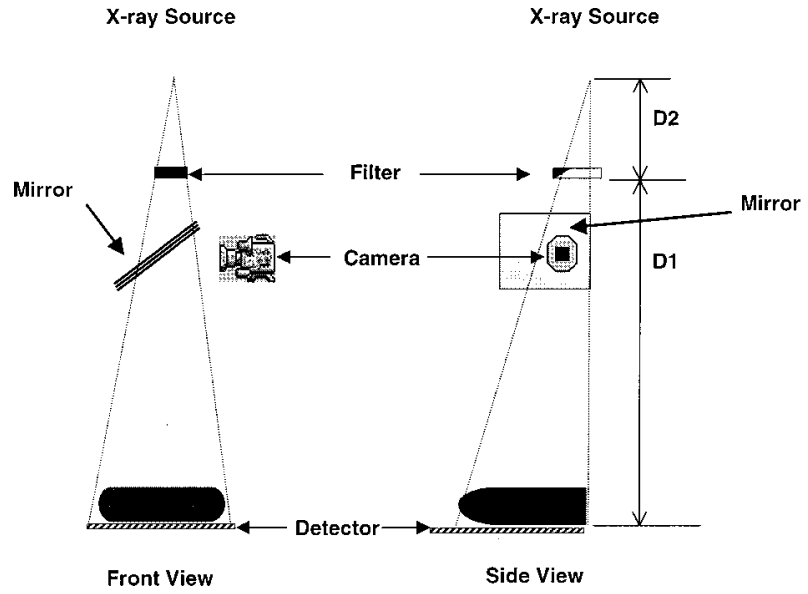

FIG. 1. Schematic of a mammography unit implemented with an equalization filter system.

filter corresponding to this group will be placed in the beam path and aligned with the breast border by a translationrotation apparatus operated under computer control. The focal-spot-to-filter distance $(D 2)$ can be varied to match different breast sizes. The mirror shown in Fig. 1 is used to acquire the $T V$ camera image and will be removed from the beam path before the acquisition of the x-ray image.

In an earlier study, Goodsitt et al. ${ }^{16}$ demonstrated that compressed breasts can be classified into a finite number of shapes and therefore only a finite number of filters are needed for equalization. In this paper, we report the results of a computer simulation study that was conducted to determine the effectiveness of using a finite number of filters in equalizing the exposures of mammograms.

\section{MATERIALS AND METHODS}

\section{A. Data set of digitized mammograms}

1004 clinical mammograms acquired with a dedicated mammographic system with a Mo anode and Mo filter were randomly selected from patient files in our department. All mammograms were recorded with Kodak Min-R/Min-RE screen-film systems. The selected images included both craniocaudal (CC) and mediolateral oblique (MLO) views. The films were digitized with a DBA Systems, Inc. (Melbourne, FL) model ImageClear M2100 film digitizer. The light sensor of this system is composed of two linear arrays of charge coupling devices (CCD) that are butted together to form a contiguous array. The original pixels in each digitized image were averaged and subsampled to produce images with a $1 \times 1 \mathrm{~mm}^{2}$ pixel size. The digitized values were later converted to 12-bit logarithmic format to yield a fairly linear relationship between the film optical density (OD) and pixel value, with larger pixel values corresponding to lower ODs. The pixel value versus OD calibration curve leveled off at an OD of about 3.6, beyond which the pixel values remained almost constant.

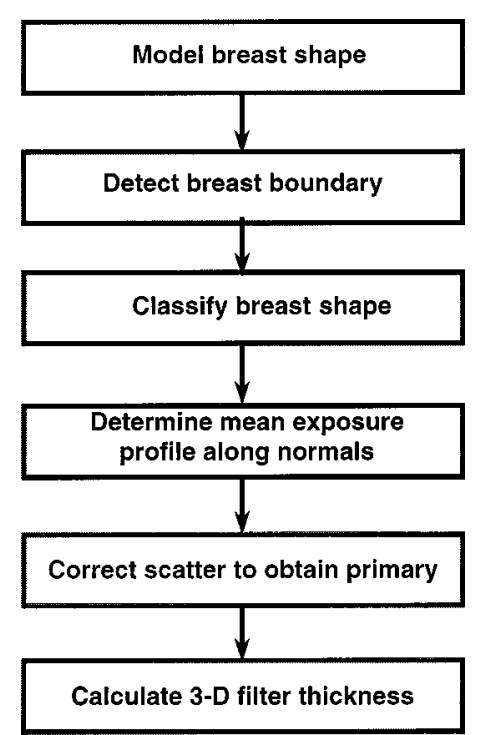

FIG. 2. Procedure for designing an external equalization filter.

\section{B. Classification of breast shapes}

An automated border-tracking algorithm was applied to the digitized images. ${ }^{18} \mathrm{~A}$ total of $470 \mathrm{CC}$ view and $484 \mathrm{MLO}$ view automatically traced borders were analyzed. This is the same set of borders that was analyzed in our previous study. ${ }^{16}$ In that study, ${ }^{16}$ we found that the breast borders could be fit very well with the polynomial $y=a x^{2}+b x^{3}$. This functional form has the advantage of producing only two coefficients $(a, b)$ which can be used in a cluster analysis to classify the border shapes. These coefficients were introduced into a $k$-means clustering algorithm. Optimal clustering was achieved for three or four groups in both $\mathrm{CC}$ and MLO views.

\section{Filter design}

Before building an actual system, we conducted a simulation study to investigate the effectiveness of the external filters. In this simulation study, we designed a simulated equalization filter for each group of breasts. The $a-b$ polynomial fit discussed earlier only describes the projected breast shape on the image. It does not account for the thickness variation near the periphery of a compressed breast. The inclusion of this third dimension in the design of the filter is the subject matter of the present study. Changes in the breast thickness at the periphery are observed as changes in the gray scale values of pixels on the digitized mammogram. Because equalization occurs in the exposure domain, the pixel values have to be converted to exposures. The conversion involves use of the digitizer calibration curve and the sensitometric curve of the screen-film system. The exposure profiles at the breast periphery estimated from the digitized mammograms for a particular group can be used to design a three-dimensional (3-D) filter for that group.

The calculation procedure used for designing an exposure equalization filter is presented as a flow chart in Fig. 2. For each image, the pixel value profiles along a number of nor- 
mals (25 to 35 ) to the automatically detected breast boundary ${ }^{18}$ were obtained. Each normal consisted of 41 points that were 1 pixel apart, of which 20 points were outside the breast and 20 were inside the breast. The 21 st point was exactly on the detected breast boundary. Thresholding criteria were employed to exclude pixels where the normals intersected lead markers or the pectoral muscle. The pixel values were converted to optical densities by using the CCD digitizer calibration curve and linear interpolation. The OD profiles along all the normals were averaged to obtain a mean OD profile for each breast. The mean OD profiles for all images in a given group were in turn averaged to obtain an average OD profile for the entire group. This process of multistep OD averaging ensured that a smooth relative exposure profile was used for filter design. This OD profile was subsequently converted to a relative exposure profile using a typical sensitometric curve for the Kodak Min-R/Min-RE screen film system.

The exposure in the profile is the total exposure including primary and scatter. The primary exposure, which the filter directly attenuates, can be obtained using the following relationship:

$$
\bar{E}_{p}=\bar{E}_{t}(1-\mathrm{SF})
$$

where $\bar{E}_{p}$ is the mean primary exposure, $\bar{E}_{t}$ the mean total exposure, and SF the scatter fraction. Dance et al. ${ }^{19}$ found in an earlier study that the scatter component of the total exposure depends on the breast composition, breast thickness, and $\mathrm{x}$-ray spectrum. However, these factors were not known for the digitized mammograms used in our study. Also, there is a variation of the scatter fraction due to the decreasing thickness in the periphery region of the breast. ${ }^{10}$ This variation depends on the thickness profile of a compressed breast and the tissue composition, which were not known. For the simulation study, we made the simplifying assumption that the scatter fraction was constant. The assumption of a constant scatter fraction causes the effect of the scatter fraction before and after equalization to cancel out. However, to keep the model complete, the scatter fraction is included in the discussion of the appropriate steps in the simulation study.

It should be noted that an actual equalization filter would effectively reduce the peak of the scatter fraction that occurs near the periphery of the breast, which was not included in our simulation. This would further improve the image quality in the peripheral region, as was shown by Lam and Chan. ${ }^{10}$

Ideally, equalization reduces the exposure at the breast periphery to approximately the same low value as that in the central region of the breast. The exposure reduction factor at a given point along a normal was therefore defined as the ratio of the minimum exposure on the normal to the relative exposure value at that point. A profile of average exposure reduction factors was generated and used to specify the filter for equalization of the breasts in a particular group.

To design a physical filter, the average exposure reduction factor profile along a normal was converted to a filter thickness profile using a filter thickness versus exposure reduction

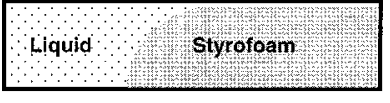

(a)

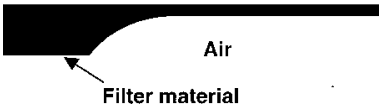

(b)
FIG. 3. Schematics of fabricating filters with (a) liquid or (b) solid material.

factor look-up table. Although the thickness matrix was not used in our simulation study, it would be useful for fabricating actual filters.

To generate a filter thickness-to-exposure reduction factor conversion table, a filter material was selected and the attenuation coefficients for this material were obtained using the $\mathrm{XCOM}^{20}$ computer program. The attenuation coefficients were then used in the following relationship to calculate the exposure reduction that could be obtained with a given filter thickness:

$$
E s_{f}\left(t_{f}\right)=\frac{\Sigma_{E} f(E) E e^{-\mu_{f}(E) t_{f}}\left(\mu_{e}(E) / \rho\right)_{\mathrm{air}}}{\Sigma_{E} f(E) E\left(\mu_{e}(E) / \rho\right)_{\mathrm{air}}}
$$

where $E s_{f}\left(t_{f}\right)$ is the filtered relative exposure, also referred to as the exposure reduction factor, $f(E)$ is the relative number of photons at a given energy $E$ of the spectrum, $\left(\mu_{e}(E) / \rho\right)_{\text {air }}$ is the mass energy absorption coefficient of air at energy $E$, and $\mu_{f}(E)$ and $t_{f}$ are the linear attenuation coefficient at energy $E$ and the thickness of the filter material, respectively. The mass energy absorption coefficients of air were obtained from Johns and Cunningham. ${ }^{21}$ This calculation provided a relative exposure of 1 when the filter thickness was zero and a value less than 1 for larger filter thicknesses. In this study, we assumed a spectrum $(f(E))$ of $\mathrm{Mo} / \mathrm{Mo} 28 \mathrm{kVp}$ with a HVL of $0.32-\mathrm{mm} \mathrm{Al} .^{22}$ The exposure reduction factor was stored along with the corresponding filter thickness as a look-up table for use in the filter design procedure.

Ideally, the energy absorbed in the screen rather than the exposure to the screen/film should be equalized. However, the energy absorption of the screen depends upon the x-ray spectrum incident on the screen. This spectrum is not known unless the imaging parameters $(\mathrm{kVp}$ and filtration), breast thickness, and composition are available, and Monte Carlo calculations are performed. For the design of an average filter for a breast group, the entire process involves a number of averaging and approximation steps. Since the goal of our approach is to reduce the exposure dynamic range rather than to produce perfect equalization, the error caused by the approximation of energy absorption in the screen by exposure will not impose a substantial effect.

We can use either solid materials, tissue-equivalent fluids or more radiodense liquids with radiation transparent molds enclosed in a sealed container to build the equalization filters. Figure 3 shows sketches of such solid and liquid filters. Examples of liquids that might be used in filters include water, saline, and water/alcohol solutions. Solid filters might be made of plastic or plastics impregnated with heavy elements. 


\section{Simulation study}

To verify the effectiveness of external-filter-based exposure equalization, the entire filtration process was simulated using computer programs and was tested on digitized mammograms. In this simulation study the filter was positioned just above the compressed breast. This simplification enabled the use of fitted curves to the breast boundary for filter alignment calculations. Since the equalization process occurred in the exposure domain, the pixel values in the digitized images were converted to relative exposure values. The exposure reductions by the filter near the breast periphery were estimated and converted to changes in pixel values. The digitized image was corrected with the appropriate pixel value change at each pixel, producing a "filtered" digitized mammogram. Changes were implemented in the pixel value domain rather than the optical density domain, because it facilitated direct display and comparison of the equalized and unequalized images on a high-resolution workstation monitor. Furthermore, because this approach did not entail printing the filtered image on film for comparison with the original film, it avoided possible artifacts in the center of the filtered breast image caused by uncertainties in the pixelvalue-to-OD conversion curve of the digitizer. Uncertainties in the pixel value changes in the peripheral region were digitally smoothed out to simulate filtration by a smooth physical filter, as detailed below. The various steps in the simulation process are presented as a flow chart in Fig. 4.

For a given mammogram to be equalized, the procedure started by detecting the boundary of the breast image using
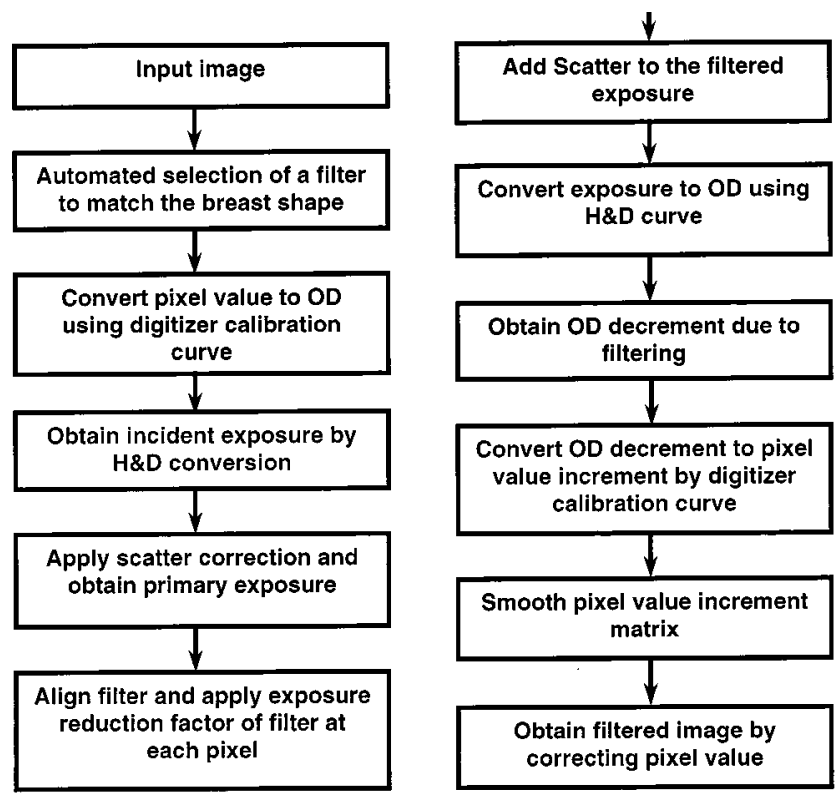

FIG. 4. Flow diagram of the simulation study for evaluation of the effects of $\mathrm{x}$-ray equalization on mammograms.

our automated breast border tracking program. The detected boundary was then fitted with the $a x^{2}+b x^{3}$ polynomial. Using the $a$ and $b$ values, the breast border was classified into a particular group. On the basis of this classification, the average equalization filter designed for that breast group was chosen. The equalization filter was represented by a 2-D ar-

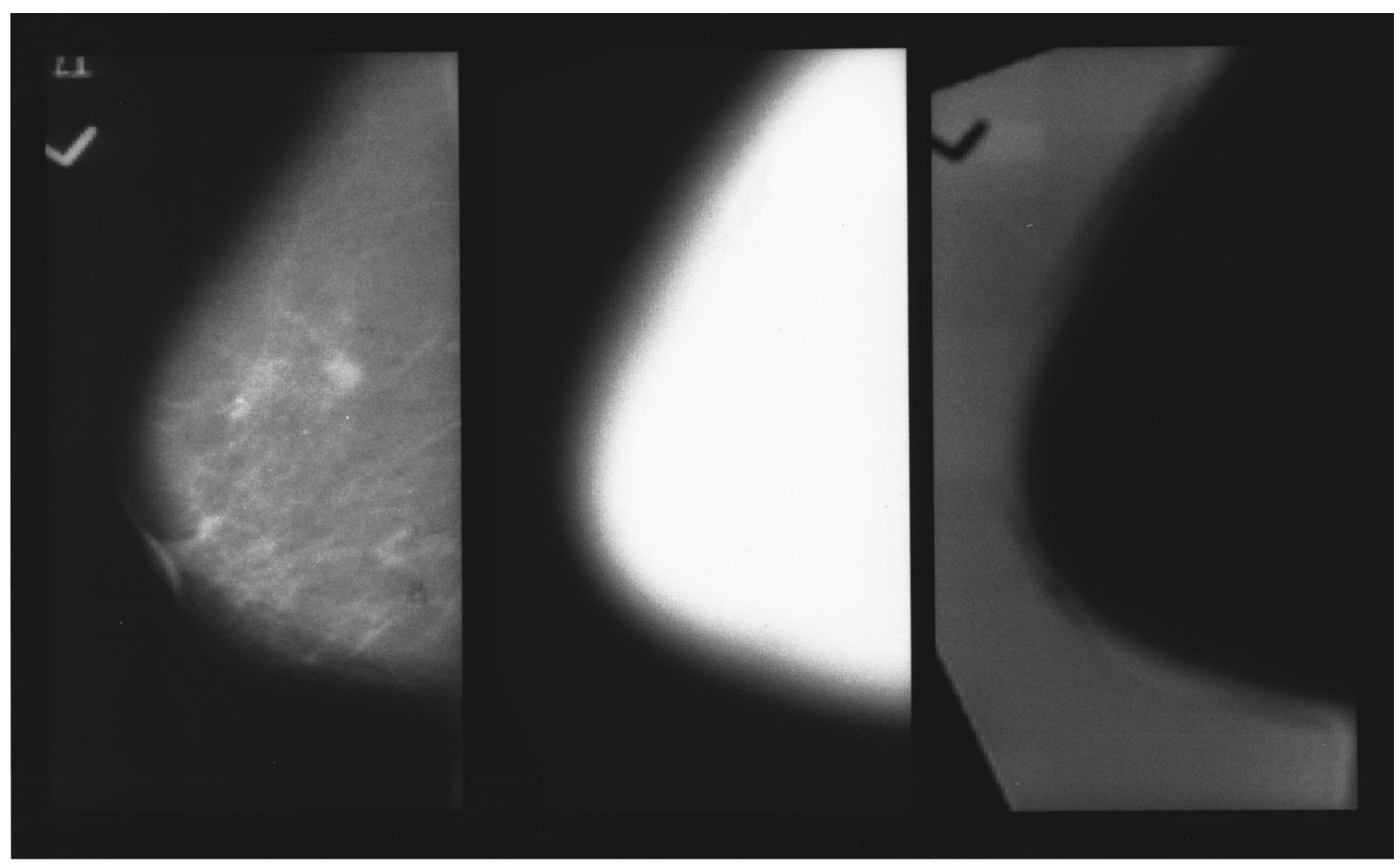

(a)

(b)

(c)

FIG. 5. An example of (a) a digitized mammogram, (b) an exposure reduction factor matrix displayed as a gray scale image, and (c) the corresponding pixel value increment matrix. 
ray of exposure reduction factors described in Sec. II C. This exposure reduction factor array was rotated and translated along both the $X$ and $Y$ directions to achieve the best alignment between the filter and the breast image. The criterion for best alignment was assumed to be the minimization of the root-mean-square (rms) distance between the filter boundary and the automatically detected breast boundary. The pixel values in the digitized mammograms were then converted to OD using the digitizer calibration curve. The $\mathrm{OD}$ at each pixel was subsequently converted to relative exposure using a typical Hunter and Driffield curve for a Kodak Min-R/Min-RE screen film system. ${ }^{23}$ The relative exposure thus obtained included both the primary and the scatter components. Since we assumed that the scatter fraction was constant over the entire breast area in this study, the total exposure would be proportional to the primary exposure. The exponential attenuation process of the filter could therefore be applied to the total exposure. The total exposure at each pixel location was reduced using the exposure reduction factor at that location. Exposure equalization by the external filter was accomplished at this step.

The equalized total exposure was subsequently converted back to OD using the H\&D curve. This OD was compared with the original OD to obtain the OD decrement for a given pixel. This OD decrement was converted to a pixel value increment using the digitizer calibration curve. Because the conversion in each step involved uncertainties, especially in the shoulder and toe regions of the H\&D curve, the 2-D array of pixel value increments contained numerical errors that would not exist with an actual filter. To reduce the fluctuations in the pixel value increments, a 2-D smoothing was performed by applying a $3 \times 3$-pixel convolution kernel to the pixel value increment array before the array was added to the original image.

Figure 5 shows an example of a digitized mammogram, the corresponding array of exposure reduction factors displayed as a gray scale image, and the array of pixel value increments also displayed as a gray scale image. The dark and gray areas in the background outside the breast indicate that the exposure reduction factor array was rotated and aligned to match the given breast shape. Additionally, the pixel value increment array is approximately a negative image of the exposure reduction factor array. The dark area within the breast region indicates pixel value increments of zero.

\section{E. Filter misalignment analysis}

We designed a Figure-of-Merit (FoM) to evaluate the “goodness of alignment" for our external-filter equalization method. First, approximately 25-35 equally spaced normals to the detected breast border were generated for each image. The pixel values in the digitized mammograms along these normals were obtained at 31 points. Only ten points outside the breast boundary were used because we were interested in the artifacts that occurred at the breast periphery. The pixel value increments used for equalization of the same image were also obtained along each normal and these values represented the 3-D profile of the filter. The pixel values decreased from the inside to the outside of the breast periphery, whereas the pixel value increments increased. The complement of the pixel value increment profile was computed by transforming the pixel value increment profile using the following relationship:

$$
\operatorname{pixcor}(i, j)=\operatorname{Max}[\operatorname{pixinc}(i)]+\operatorname{base}(i)-\operatorname{pixinc}(i, j),
$$

where $\operatorname{pixcor}(i, j)$ was the transformed pixel value increment, base $(i)$ was the minimum pixel value increment on the $i$ th normal and was obtained by averaging the pixel value increments over the last ten points inside the breast along the $i$ th normal, Max $[\operatorname{pixinc}(i)]$ was the maximum pixel value increment on the $i$ th normal, and pixinc $(i, j)$ was the pixel value increment at the $j$ th point along the $i$ th normal.

The correlation coefficient between the pixel value profile and the transformed pixel value increment profile was defined as

$$
\operatorname{corf}(i)=\frac{\sum_{j}(\operatorname{pix}(i, j)-\operatorname{mpix}(i))(\operatorname{pixcor}(i, j)-\operatorname{mpixcor}(i))}{\left[\sum_{j}(\operatorname{pix}(i, j)-\operatorname{mpix}(i))^{2}\right]^{1 / 2}\left[\Sigma_{j}(\operatorname{pixcor}(i, j)-\operatorname{mpixcor}(i))^{2}\right]^{1 / 2}},
$$

where $\operatorname{pix}(i, j)$ and $\operatorname{mpix}(i)$ indicate the $j$ th pixel value and the mean pixel value along the $i$ th normal in an unfiltered image. The values $\operatorname{pixcor}(i, j)$ and $\operatorname{mpixcor}(i)$ are the transformed pixel value increment and the mean transformed pixel value increment, respectively, along the same $i$ th normal. The summation $j$ was over all 31 points along the $i$ th normal. The correlation coefficients for all the normals in a given image were averaged and a mean correlation coefficient was obtained. This mean correlation coefficient was used as the FoM that quantified the match between the filter and the breast image. A good match between the transformed pixel value increment profile and the pixel value profile in the original image would result in an FoM close to 1.

To study the sensitivity of the FoM to misalignment artifacts in an equalized image, we simulated some situations of misalignment by displacing the filter either laterally or transversely. The simulated filter was displaced from its optimal position (Sec. II D) by 2, 4, and $6 \mathrm{~mm}$ ) in either direction, and the corresponding equalized images were generated. By inspecting the changes in the FoM and the misalignment artifacts on the equalized images at different amounts of filter displacement, we could judge if the FoM could be corre- 


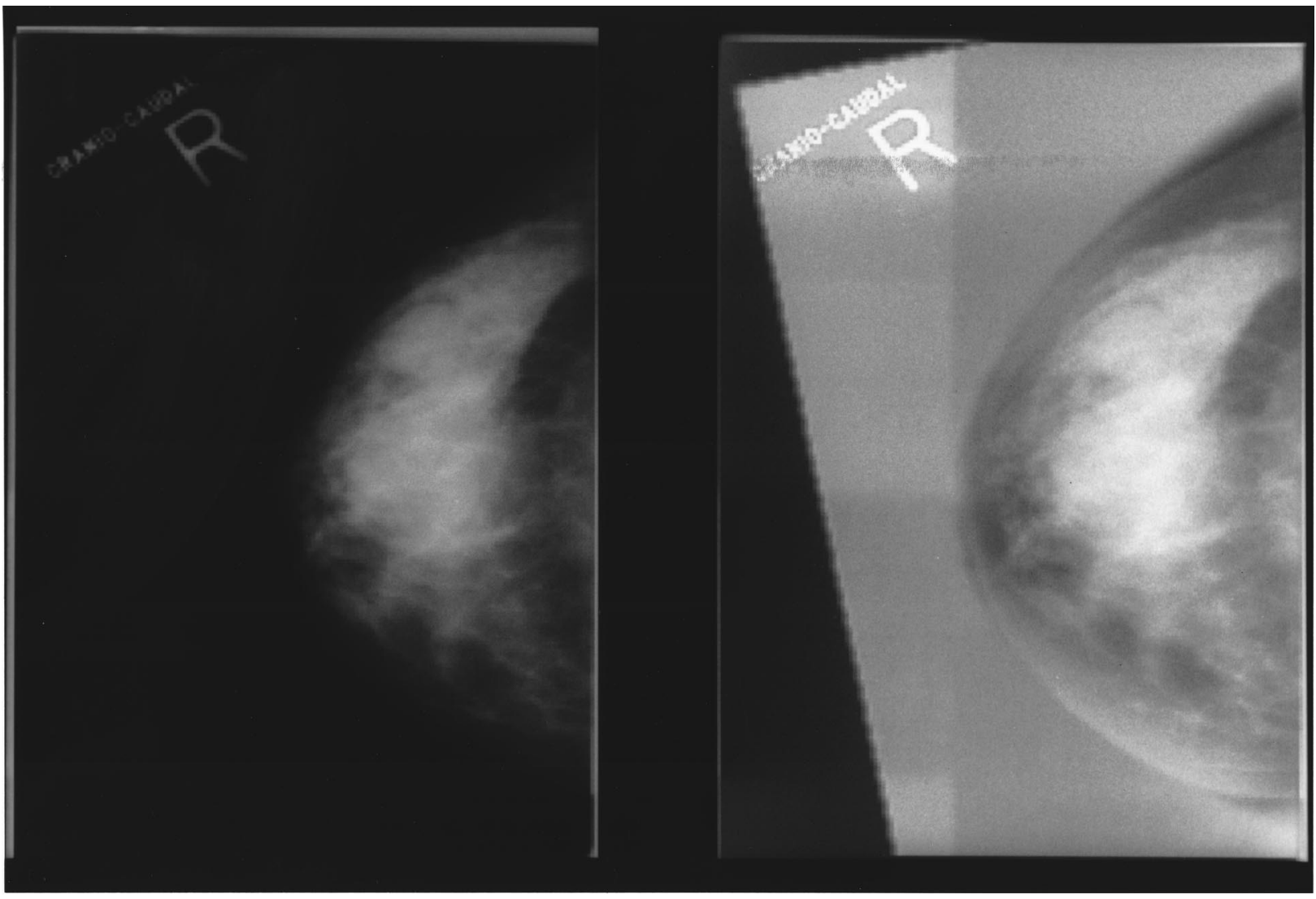

(a)
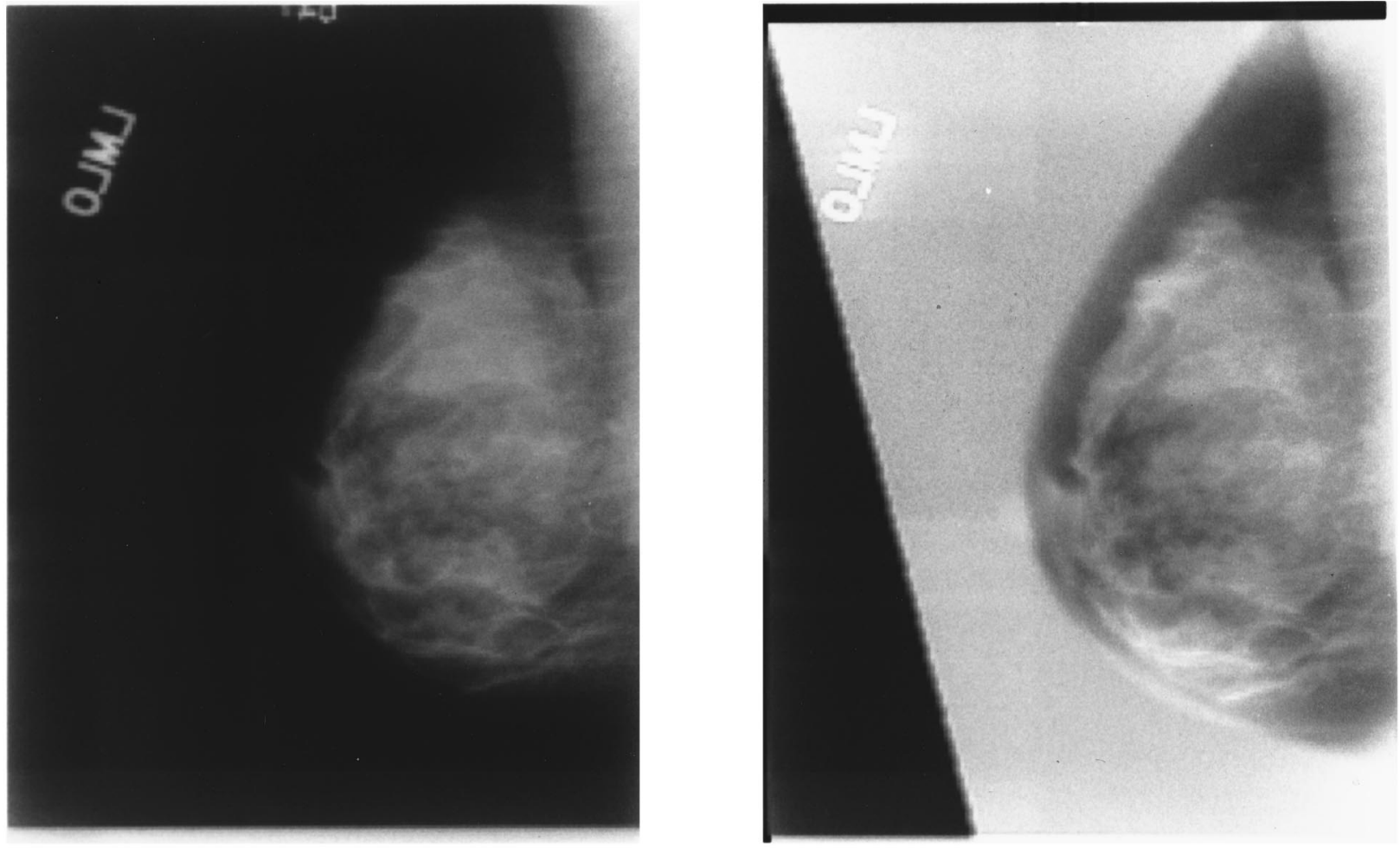

(b)

FIG. 6. Examples of (a) unequalized and equalized $\mathrm{CC}$ view images and (b) unequalized and equalized MLO view images. 


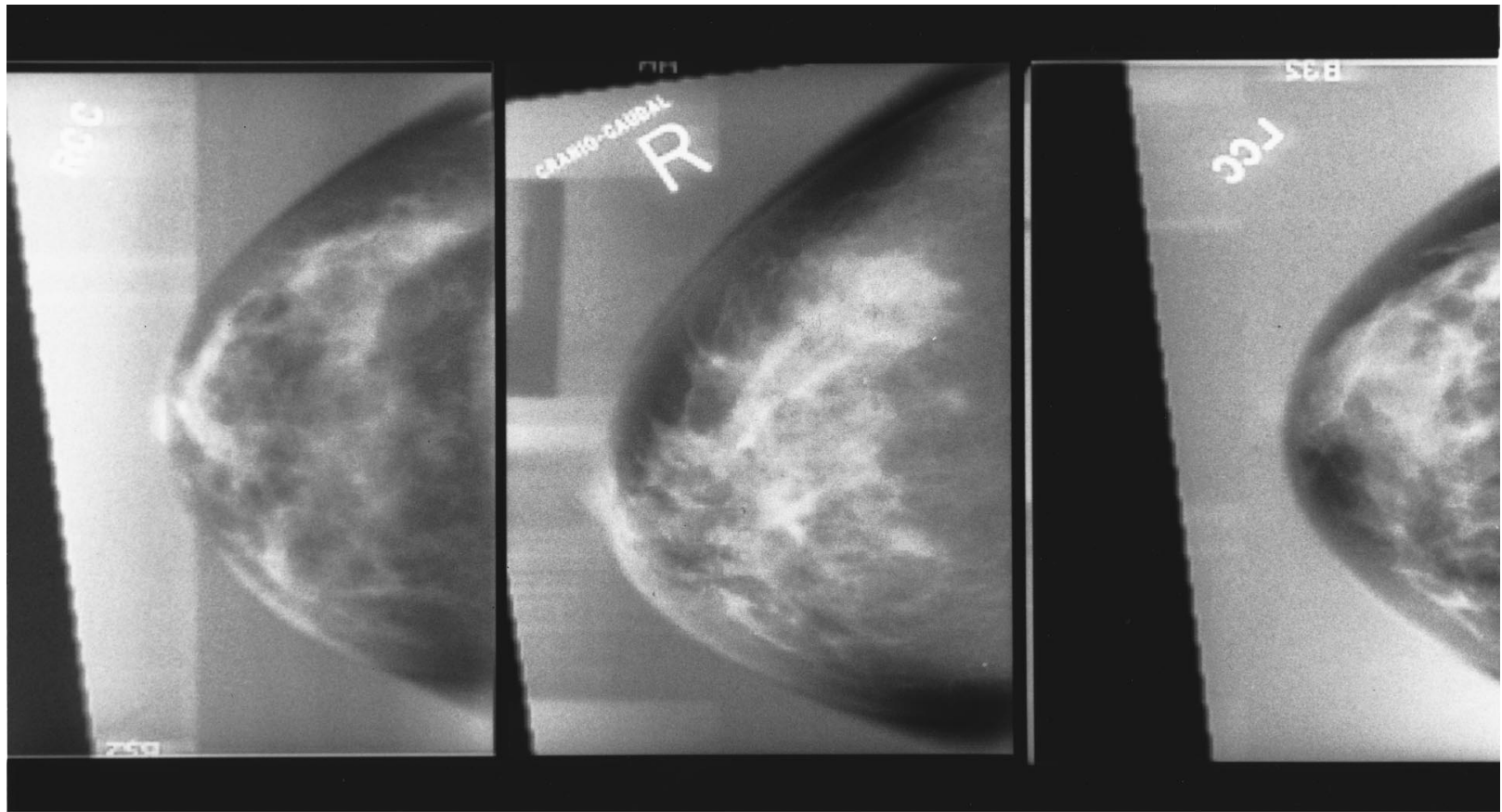

(a)
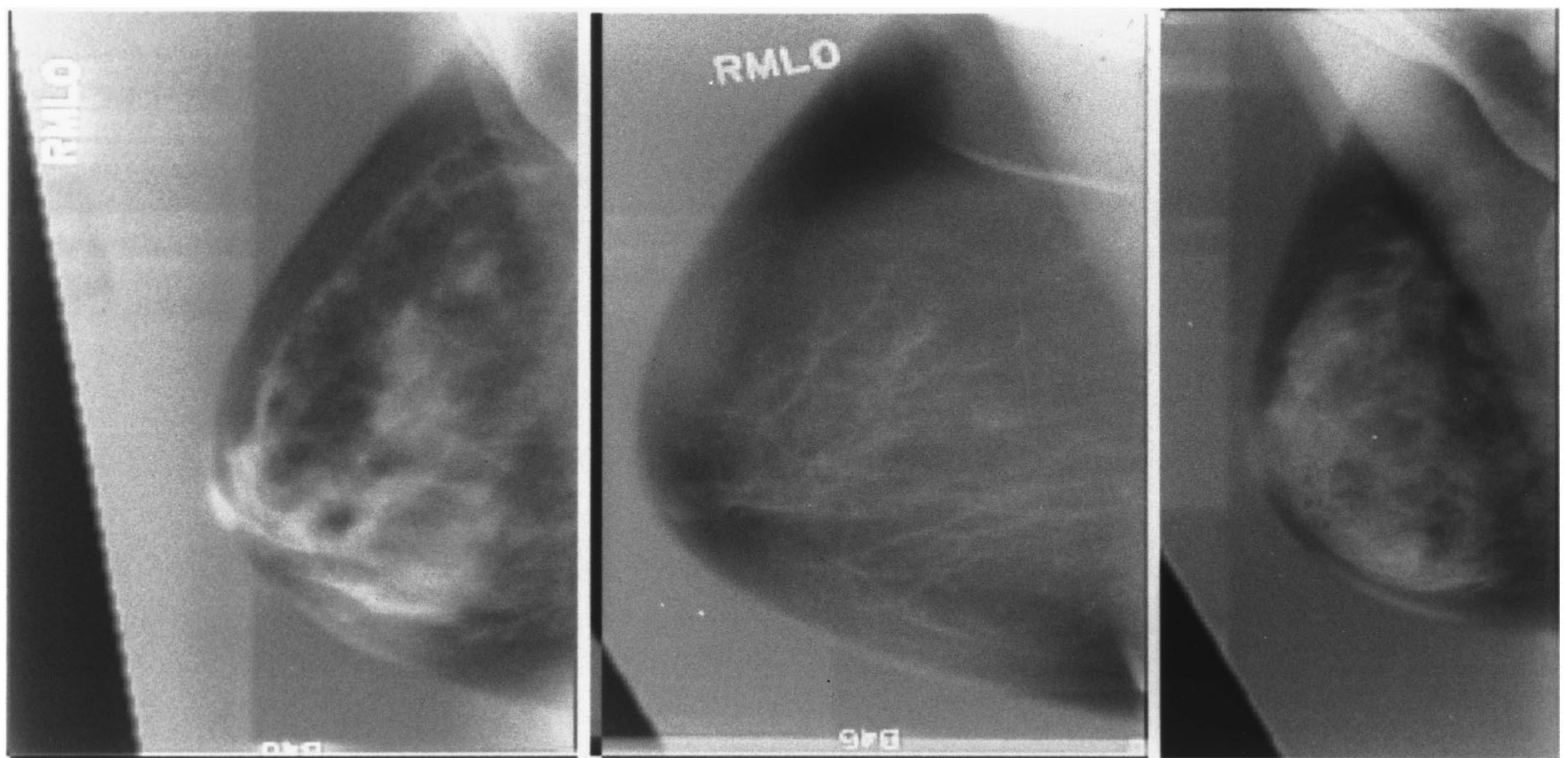

(b)

FIG. 7. A set of (a) CC view and (b) MLO view images obtained using external exposure equalization technique. These images used an average filter specific to their group. All the CC view images are from the same group. Similarly, all the MLO view images belonged to a single group.

lated with the goodness of filter alignment. We also analyzed the histogram of the FoMs for the CC view and MLO view group of images at $\pm 2 \mathrm{~mm}$ filter displacements. This analysis provided an estimate of the tolerance requirement for the construction of an automated filter alignment device.

\section{F. Observer study}

The misalignment analysis described above provided a mathematical relationship for evaluating the match between the filter profile and the exposure profiles at the breast periphery; however, it did not consider some of the subjective image quality preferences of a human observer.

To test the effectiveness of the filter, 60 images from the $\mathrm{CC}$ view group were randomly selected and were viewed by an experienced radiologist. The quality of the equalized image was ranked between 1 and 5. A quality rating of 1 represented an image that was significantly degraded by artifacts caused by equalization, and a quality rating of 5 represented an image that exhibited near perfect equalization. The radiologist also rated the breast densities in terms of the ACR-BIRADS categories 1 to 4, where 1 signified almostentirely fatty and 4 signified extremely dense. 


\section{G. Preliminary phantom experiment}

To demonstrate the effect of an external filter, we built two types of filters and obtained equalized images of a breast phantom. A $4.5 \mathrm{~cm}$ CIRS (CIRS, Inc., Norfolk, VA) phantom of $50 \%$ glandular and $50 \%$ adipose composition was chosen to represent an average compressed breast.

The first type of filter was a liquid filter. It consisted of a handcrafted Styrofoam mold and a container filled with saline $(\approx 3 \%$ of $\mathrm{NaCl}$ by weight). The size of the Styrofoam mold was calculated by minifying a full scale drawing of the CIRS phantom. A geometric minification factor of 3 was assumed for this calculation. Two paper templates with different minification factors were made to represent the top and the bottom surfaces of the filter mold. These two pieces were pasted on to an approximately $2 \mathrm{~cm}$ thick Styrofoam sheet and the filter mold was then carved out using the paper templates as a guide. When the container was filled, the thickness of water was $2 \mathrm{~cm}$ outside the mold and it gradually decreased to less than $1 \mathrm{~mm}$ over the top of the mold. Care was taken to make sure that the liquid layer over the mold covered the entire field of view. This design ensured that there was minimal beam hardening over the central region of the breast and there were no artifacts due to a discontinuity of the liquid filter material at the breast boundary.

The second type of filter was a solid filter. It consisted of a piece of Teflon attached to a thin Plexiglas plate. The Plexiglas plate was used to protect the thin edge of the Te-

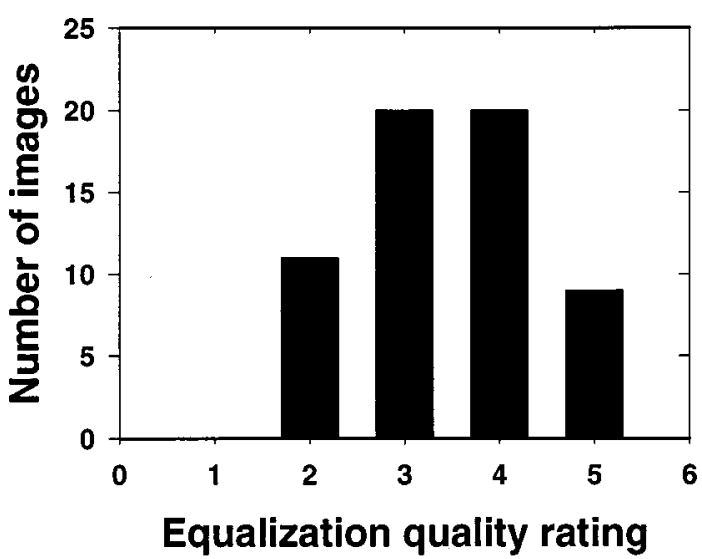

FIG. 8. Distribution of equalization quality ratings for 60 equalized images, evaluated by an experienced breast radiologist.

flon filter. The solid Teflon piece was machined using a computer-controlled mill to a wedge shape that approximated an equalization filter. The Teflon filter is a $5 \mathrm{~cm}$ wide rectangular strip that has a maximum thickness of $1.8 \mathrm{~cm}$. As a prototype, it was not fabricated to match a breast shape in the $X-Y$ plane.

For each filter, the filter-to-phantom distance and the filter orientation were adjusted manually to obtain the best alignment between the filter and the breast phantom to acquire an exposure-equalized image.

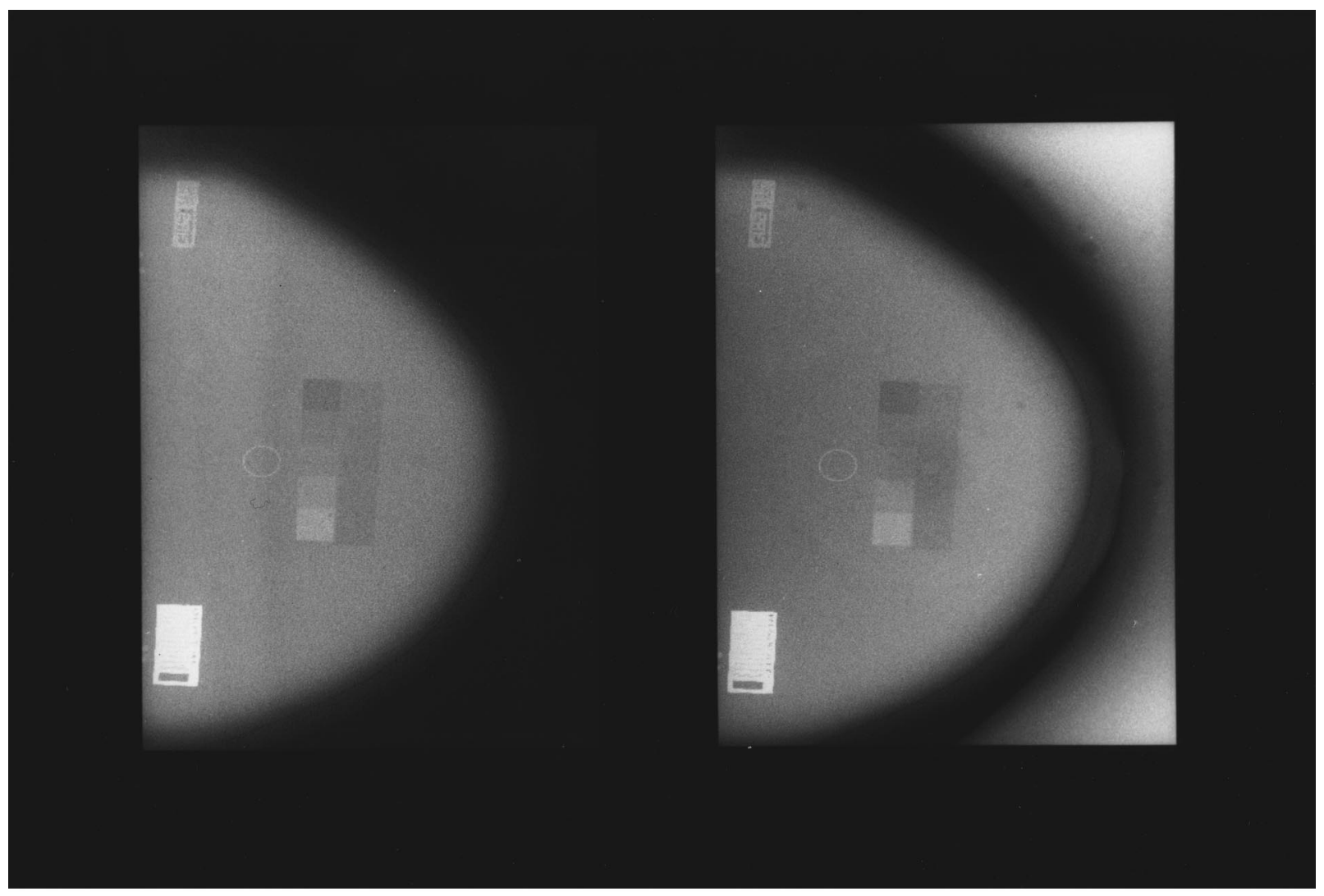

(a)

(b)

FIG. 9. The CIRS phantom image (a) unequalized and (b) equalized with a liquid filter. 


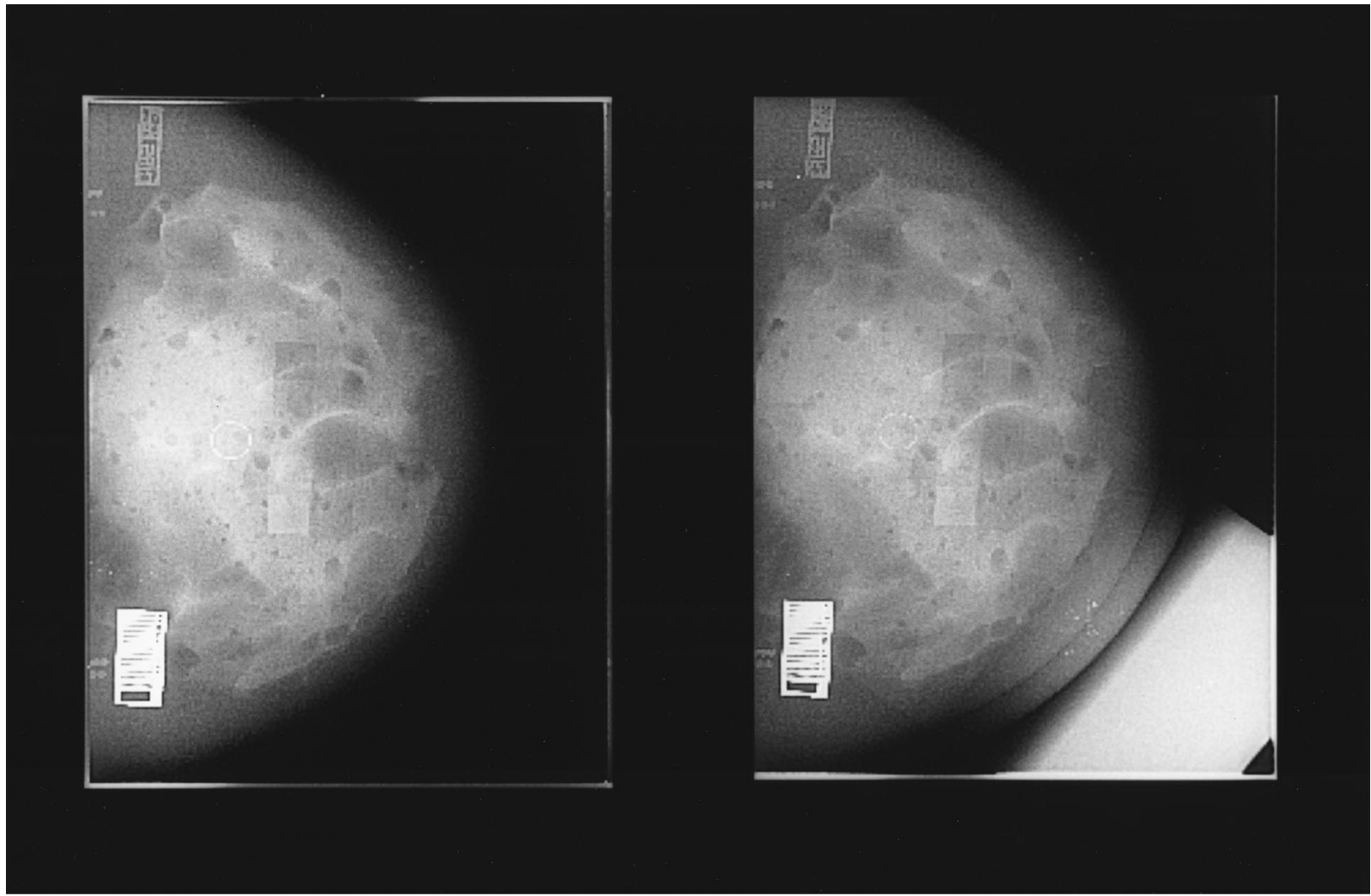

(a)

(b)

FIG. 10. The CIRS phantom image (a) unequalized and (b) equalized with a solid filter made with Teflon. A piece of silly putty was placed on top of the phantom to simulate dense breast parenchyma and calcium carbonate specks were placed at the periphery. The visibility of the peripheral region of the dense parenchyma and of the breast phantom is improved by equalization.

\section{RESULTS}

\section{A. Simulation study}

The digitized mammograms in the CC and MLO views were each classified into three groups. ${ }^{16}$ To have reasonable statistics, we chose the largest group in each view as case samples for our simulation study. The selected CC view group contained 196 images and the MLO view group contained 171 images. For each of these groups, we estimated the mean exposure range as the ratio of the maximum to the minimum exposures along the average relative exposure profile (described in Sec. IIC). These exposure ranges were found to be 5.46:1 and 5.18:1 for CC and MLO views, respectively. These exposure ranges are lower than what we would expect for breast images and will be discussed further in the Sec. IV. Using the average exposure reduction factor array and the steps described in Sec. IID, the pixel value increment array and the equalized image were computed for each mammogram in the group. Figure 6 shows examples of the unequalized and equalized images from the $\mathrm{CC}$ and the MLO groups. Figure 7 shows additional examples of equalized images from the CC and MLO view groups.

\section{B. Observer study}

A histogram of the equalization quality rating by the radiologist is shown in Fig. 8. It can be seen that about $81 \%$ of the images had ratings greater than or equal to 3 . The radiologist rated 6 images to be in ACR-BIRADS breast density category 1,31 to be in category 2,16 to be in category 3 , and 7 to be in category 4 .

\section{Preliminary phantom experiment}

The images of the CIRS phantom before and after equalization with saline as a filter material are shown in Fig. 9. It can be seen that the filter equalized the periphery region around the nipple reasonably well. However, the filter undercompensated the periphery in the upper and lower parts of the image. This was probably caused by the mismatch between the filter boundary and the breast border in these regions because the shape of the handcrafted mold did not match very well with the breast phantom shape in the $X-Y$ plane. The 0.4-cm-thick fatty skin layer in the CIRS phantom is clearly visible in the equalized image near the nipple region. Some dark spots seen in this image were due to air bubbles entrapped near the mold.

Images of the CIRS phantom before and after equalization with the Teflon filter are shown in Fig. 10. A piece of silly putty was added on the phantom to simulate dense parenchyma in a breast. The filter strip was oriented approximately in the 4 o'clock direction in the equalized image. The fatty skin layer in the CIRS phantom and some calcium car- 
TABLE I. The effect of $2 \mathrm{~mm}$ filter displacement on the FoM for the CC and MLO view images.

\begin{tabular}{lccccc}
\hline \hline \multirow{2}{*}{$\begin{array}{c}\text { Filter } \\
\text { displacement }\end{array}$} & \multicolumn{2}{c}{ CC view group } & & \multicolumn{2}{c}{ MLO view group } \\
\cline { 2 - 3 } \cline { 5 - 6 } & Mean FoM & $\begin{array}{c}\text { \% of Images } \\
\text { FoM } \leqslant 0.8\end{array}$ & & Mean FoM & $\begin{array}{c}\text { \% of Images } \\
\text { FoM } \leqslant 0.8\end{array}$ \\
\hline $0 \mathrm{~mm}^{\mathrm{a}}$ & 0.89 & $6 \%$ & & 0.85 & $19 \%$ \\
Transverse $+2 \mathrm{~mm}$ & 0.87 & $12 \%$ & & 0.83 & $29 \%$ \\
Transverse $-2 \mathrm{~mm}$ & 0.90 & $8 \%$ & & 0.86 & $16 \%$ \\
Lateral $+2 \mathrm{~mm}$ & 0.88 & $12 \%$ & & 0.84 & $24 \%$ \\
Lateral $-2 \mathrm{~mm}$ & 0.90 & $5 \%$ & & 0.85 & $16 \%$ \\
\hline
\end{tabular}

${ }^{\mathrm{a}}$ Filter location determined by minimum rms criterion.

bonate specks placed near the periphery are clearly visible in the region equalized by Teflon filter. The contrast of the edge of the simulated dense parenchymal region is also greater in the equalized part of the image.

\section{Misalignment analysis}

Histograms of the FoMs for the CC and MLO view images at the optimal filter position are plotted in Fig. 11. It can be seen that the mean FoM for the group of $\mathrm{CC}$ view images was 0.89 and over $93 \%$ of the images had FoMs greater than 0.8. Only 12 of the 196 images had FoMs less than or equal to 0.8 and the minimum FoM was 0.72. Similarly, for the group of MLO view images the mean FoM was 0.85 and about $81 \%$ of the images had FoMs greater than 0.8 . Only 33 of the 171 MLO images had FoMs less than or equal to 0.8 . Thus, the average filter shape matched well with over $80 \%$ of the images in a given group.

The distribution of the FoMs at $\pm 2 \mathrm{~mm}$ filter displacement was also analyzed. The mean FoM and the percentages of images that had FoM values less than or equal to 0.8 are tabulated in Table I. Equalized images with $0 \mathrm{~mm}$ and \pm 6 $\mathrm{mm}$ displacements of the filter in the lateral and transverse directions are shown in Fig. 12. The FoMs that were calculated for each image are also shown. For this example, the rms difference between the breast border and the filter border at the "optimal" alignment position $(0,0)$ was $2.13 \mathrm{~mm}$ and the FoM was 0.89 . There is a dark boundary around the upper section of the breast border due to the slight misalignment as indicated by the rms error. Displacing the filter by 2 $\mathrm{mm}$ in the negative lateral direction (not shown in Fig. 12) reduced the dark rim in the upper border section to lower OD and the FoM increased to 0.90. As the filter was displaced transversely away from breast, the FoM reduced to 0.87 , 0.82 , and 0.77 for 2,4 , and $6 \mathrm{~mm}$ displacements, respectively. The OD along the breast periphery increased as the transverse displacement increased positively, indicating increasing under-compensation by the filter. On the other hand, when the filter was displaced in the negative transverse direction, the breast periphery became increasingly overcompensated and the FoM reduced to $0.86,0.84$, and 0.79 for 2, 4, and $6 \mathrm{~mm}$ displacements, respectively. Except for the $-2 \mathrm{~mm}$ displacement discussed above, a similar reduction in FoM was also observed for filter displacements along the lateral direction. This example illustrates the effectiveness of the correlation coefficient in quantifying the degree of misalignment.

\section{DISCUSSION}

\section{A. Effectiveness of external exposure equalization method}

It can be seen in Fig. 6 that the equalization along the breast periphery is very good in these examples, despite the fact that the filter was designed by using an average exposure profile for a given breast shape class. It can also be seen from Fig. 7 that although the sizes and shapes of the breasts varied over a wide range, the average filter was effective in equalizing these images without obvious artifacts. Digitizer artifacts due to the unbalanced sensitivity of the two CCD arrays, charge transfer, and blooming can be seen on some of the images. The digitizer artifacts are specific to this simulation study and will not exist in an actual equalization pro-

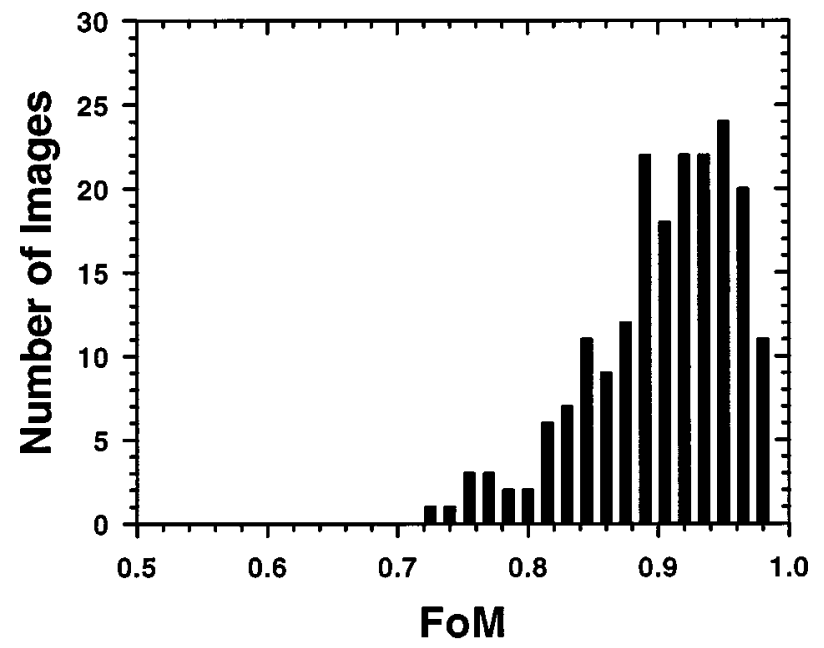

(a)

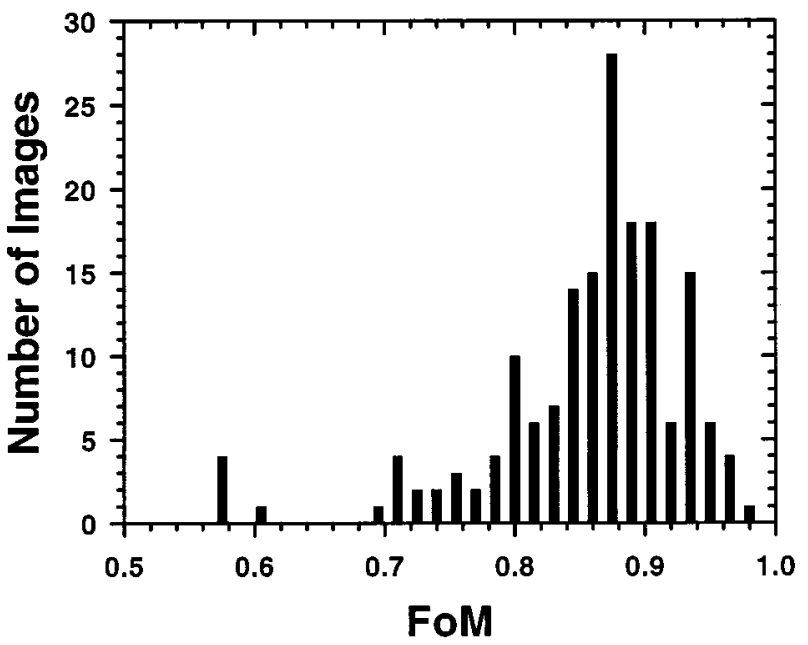

(b)

FIG. 11. Distribution of the Figure-of-Merit for the group of (a) CC view and (b) MLO view images. 


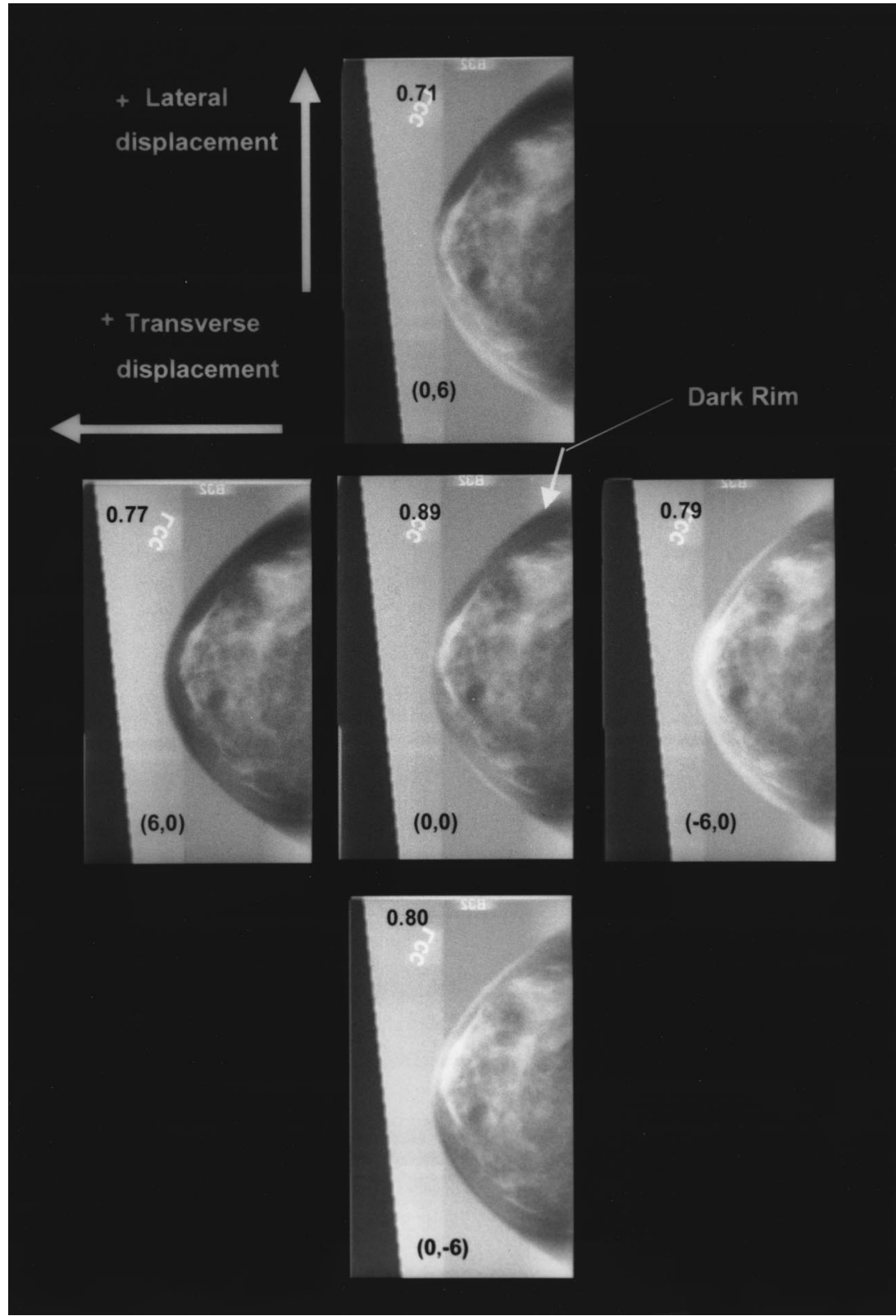

FIG. 12. Effect of filter misalignment on the FoM. The dark areas seen along the breast periphery are due to under-compensation whereas the lighter areas are due to over-compensation. The numbers in parentheses indicate the filter displacements and the numbers at the upper left corner are the corresponding FoM values.

cess. These examples illustrate that a small number of external filters can be designed to provide effective equalization at the breast periphery for breasts in a given mammographic view.

\section{B. Misalignment and filter positioner design}

The results in Table I indicate that a $2 \mathrm{~mm}$ displacement did not reduce the FoM substantially for the $\mathrm{CC}$ view images. The fraction of CC images with FoMs greater than 0.8 was over $88 \%$ for $2 \mathrm{~mm}$ displacements in all directions. For the MLO view images, the mean FoM for $2 \mathrm{~mm}$ displacements in all directions was still greater than 0.8. However, the fraction of images with FoMs greater than 0.8 decreased to a minimum of $71 \%$. Because the shapes of MLO images are more complicated than $\mathrm{CC}$ view images, the simple polynomial used for the filter shape probably cannot tolerate misalignment as well as in a $\mathrm{CC}$ view. This may have to be improved through shaping of the filter thickness profile so that less equalization is performed at the pectoral muscle and lower breast regions, or through a more complicated filter shape. The latter approach may increase the number of filters required for the MLO view.

From the filter displacement simulation, we estimated that a misalignment of up to $2 \mathrm{~mm}$ between the filter edge and breast boundary is tolerable for the $\mathrm{CC}$ view. However, for the MLO view, the misalignment may have to be less than 1 $\mathrm{mm}$. Because the simulation study used digitized mammograms and an analytical filter, the misalignment occurred in the image plane. In actual implementation the filter will be about $20 \mathrm{~cm}$ from the focal spot. With such a geometry, the $1 \mathrm{~mm}$ corresponds to about $0.3 \mathrm{~mm}$ in the filter plane. Such a tolerance is achievable through commercially available $X-Y$ translators. Moreover, placement of the filter $20 \mathrm{~cm}$ from the focal spot will result in significant geometric blur of 
the projected filter shape, which should further relax the requirements for filter alignment. Thus, we expect that it will be feasible to build an automatic filter selection and alignment system economically.

\section{Observer study}

Although the data set used in this observer study was small, the radiologist's quality ratings (Fig. 8) demonstrate that the image quality for most of the equalized images was greater than or equal to 3, which can be considered acceptable. The average quality rating was 3.3 for images in breast density categories 1 and 2, and it was 4.1 for images in breast density categories 3 and 4 . The lower average quality rating for the images with lower breast density is probably caused by the fact that they are more likely to be overcompensated by the average filter. This is consistent with the radiologist's comments after the observer study that (1) slight under-compensation and images with a dark rim are in general preferred, (2) any over-compensation and equalized breast images with a low-OD boundary are undesirable, and (3) small fatty breasts do not require equalization.

\section{Discrepancy in the estimated exposure dynamic range of breast images}

The average exposure range values presented earlier were lower than an expected range of 20:1 for a typical mammogram. To understand this discrepancy, histograms of maximum exposure ranges in each image for the CC and MLO view image groups were generated. The maximum exposure range in an image was estimated as the ratio of the maximum to the minimum exposures among all exposure profiles along a set of normals in that image. The histograms of the maximum exposure ranges are shown in Fig. 13. The mean values for the CC and MLO view histograms are 10.24 and 9.97, respectively, which are still lower than the expected dynamic ranges of breast images. A major reason for the underestimation of the exposure range in the digitized mammogram is the inability of the film digitizer to digitize accurately in the high-OD region. The OD-to-pixel value calibration curve of the digitizer leveled off at about 3.6 OD, whereas the maximum OD of mammography film can be greater than 4 . Any OD above 3.6 essentially was digitized to about the same pixel value. The OD converted from a pixel value in the breast periphery therefore could be greatly underestimated. This problem was further amplified by the small gradient in the shoulder region of the H\&D curve; a small error in OD corresponded to a large error in relative exposure. Another reason for the small exposure range from our estimation is that searching for the minimum exposure along the selected normals did not guarantee that the densest region in the breast image would be found. The extreme dynamic range of breast images is usually estimated from the densest region (OD near fog and base level) relative to the maximum exposure outside the breast region.

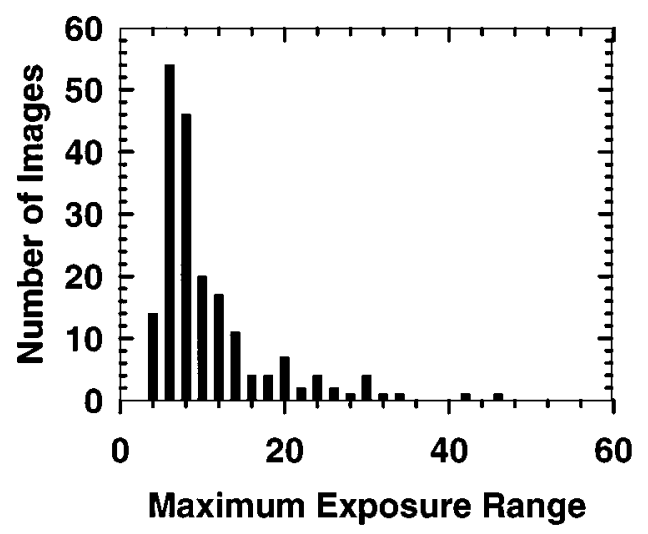

(a)

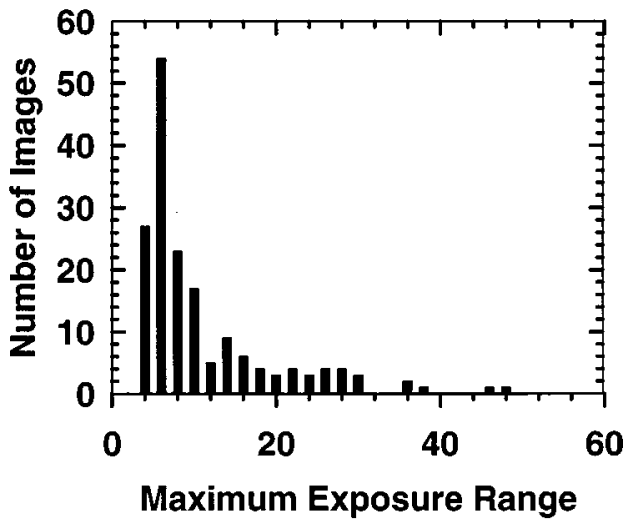

(b)

FIG. 13. Histogram of maximum exposure range for the group of (a) CC view and (b) MLO view images.

\section{E. Drawbacks of the external exposure equalization method}

The equalization filter may cause artifacts on some images. These artifacts are mainly due to mismatches between the equalization filter and the breast.

Part of the edge or the entire edge may appear brighter than the surroundings. This is an artifact of overcompensation, which arises when part of the filter or the entire filter extends too far into the breast and/or the slope of the filter is greater than that needed to compensate for the exposure gradient at the periphery of the breast. Overcompensation may result in contrast reduction because of the low gradient in the toe region of the H\&D curve.

A dark rim or dark areas may appear around the breast. This is an artifact caused by under-compensation. It arises when part of the filter or the entire filter is positioned too far outside the breast or when the slope of the filter is less than that needed to compensate for the exposure gradient at the periphery of a particular breast. Under-compensation is likely to be more acceptable to radiologists because the breast periphery is improved even if it is not completely equalized. This is confirmed by the impression of the radi- 


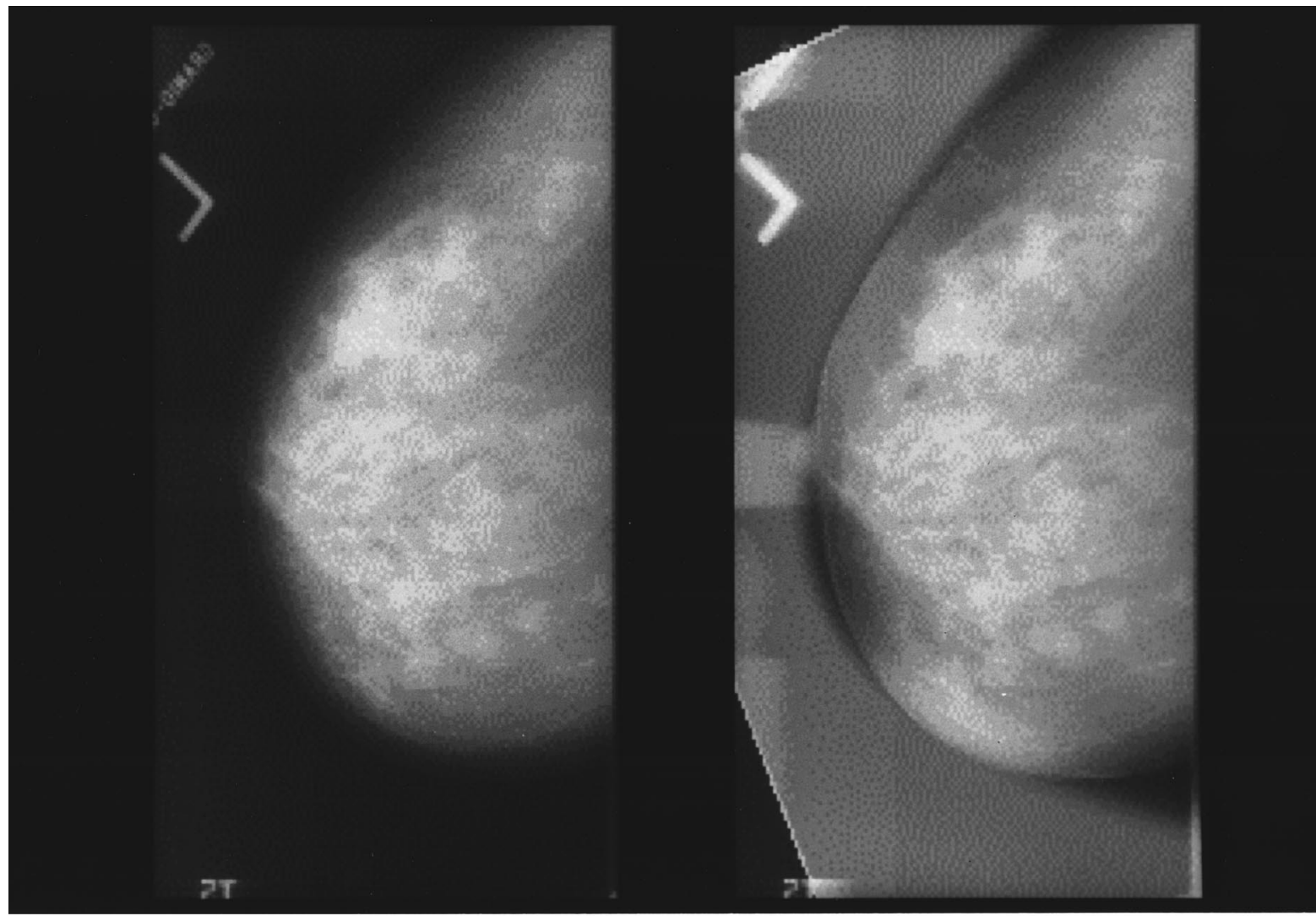

(a)

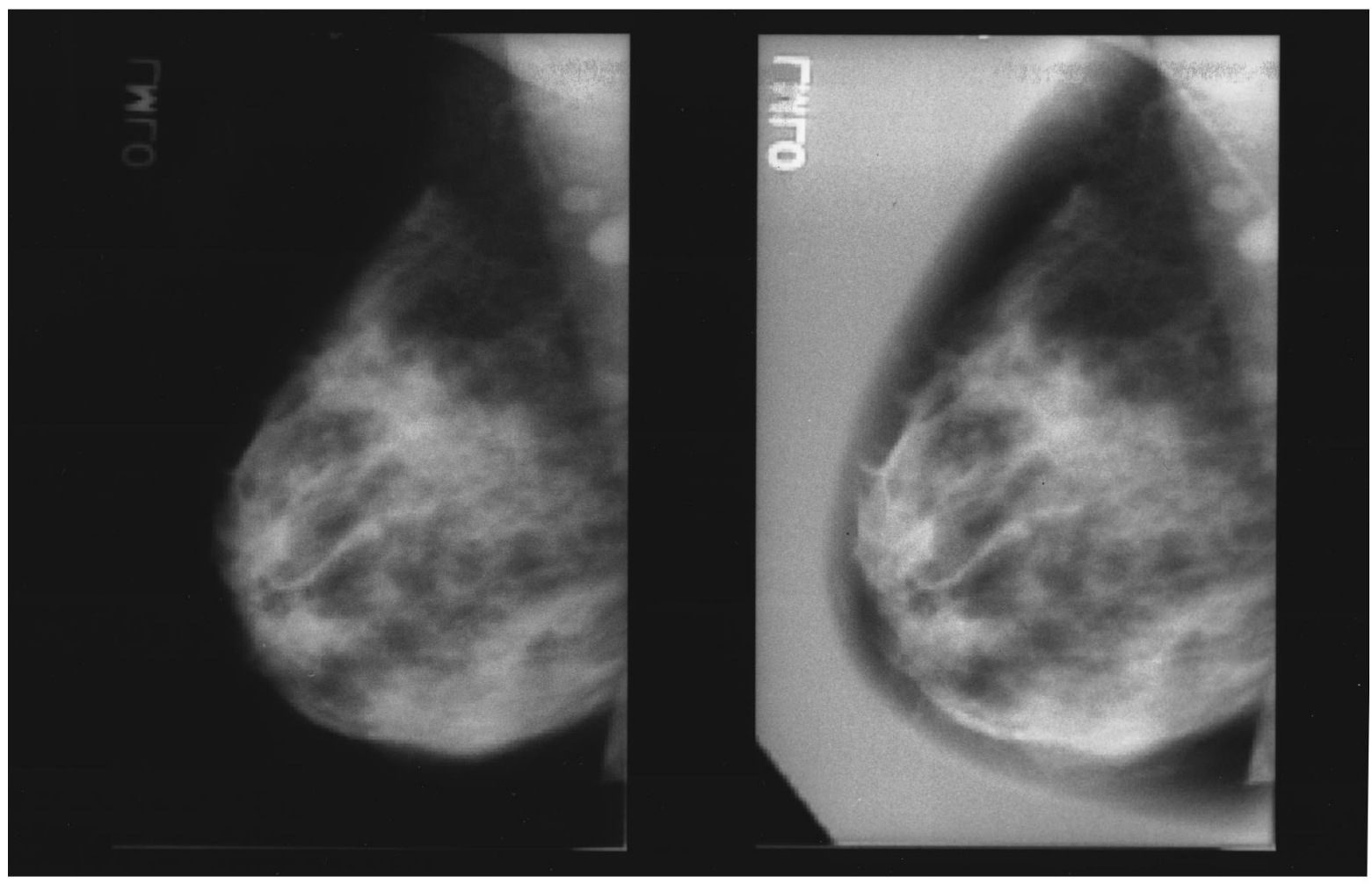

(b)

FIG. 14. Example of images with artifacts: (a) unequalized CC view image (left) and equalized image with under-compensation artifact (right) in the upper portion and below the nipple and (b) unequalized MLO view image (left) and equalized image with over-compensation artifact (right) in the lower portion of the breast periphery. 
ologist who evaluated the equalized images in the observer study.

Figure 14 shows examples of images from the $\mathrm{CC}$ and MLO groups that demonstrate some of these artifacts.

One problem revealed by the simulation study of the equalization system is that a single filter thickness for all breasts in one group may not be adequate. It is known that breast density and thickness do change the amount of exposure compensation required for equalization. The rightmost image in Fig. 7(a) shows that the image background is brighter than the breast, indicating that the filter was thicker than that needed for this breast. The rightmost image in Fig. 7(b) shows an under-compensated image with a background darker than the breast, which indicates that the filter was thinner than that needed for this breast. In actual implementation, the mismatch due to breast thickness can be reduced by building three filters with different thicknesses for each breast shape group; one of these filters may be selected depending on the breast thickness. A criterion can also be set so that no equalization will be used for breasts thinner than a threshold thickness. Although the number of filters will increase by threefold, the total number of filters is still reasonably small and may be acceptable.

For this simulation study, we used a single exposure profile to generate the filter profile along the entire breast border. It can be seen from Fig. 7(b) that, in equalized MLO view images, the pectoral muscle regions are generally overcompensated relative to the breast region. This result indicates that a uniform filter profile along the entire breast border is not a good approximation for the MLO view images. A solution to this problem may be making the filter thickness gradually taper off in the pectoral muscle region for the MLO groups.

\section{F. Implementation of an exposure equalization system}

The simulation study used a digitized mammogram as the input image. However, in actual implementation a digital video image of the compressed breast will be used for selection and alignment of the filter. A color video image of the compressed breast can be acquired with a dark green background to facilitate segmentation and breast shape classification. Experiments in our laboratory have shown that compressed body parts have a higher red component. A red component image of the compressed breast can be segmented easily from a dark green background by simple thresholding. The breast shape as determined from the segmented boundary may need corrections for potential differences in the geometry of the video and $\mathrm{x}$-ray images. The algorithms developed here for the simulation study can then be used for breast shape classification, filter selection, and alignment of the filter with the breast boundary.

\section{CONCLUSIONS}

We have demonstrated the effectiveness of an exposure equalization technique for mammographic imaging using a small set of near patient-specific external filters. Through a computer simulation study on a large set of digitized $\mathrm{CC}$ and MLO view breast images, it is shown that an average filter for a group of breasts with a similar shape can be designed using a polynomial to represent the breast shape and an average exposure profile to derive the filter thickness profile. This average filter is effective in providing exposure equalization without significant misalignment artifacts for approximately $80 \%$ of the images used in this study. Additionally, it is shown that slight misalignment of the filter is tolerable.

A preliminary phantom study using handcrafted filters indicates that it is feasible to fabricate an external exposure equalization filter using either a solid filter material or a negative mold with a liquid filter material.

\section{ACKNOWLEDGMENTS}

This work was supported by Grant No. DAMD 17-94-J4292 from the U. S. Army Medical Research and Material Command. The content of this publication does not necessarily reflect the position of the government and no official endorsement of any equipment and product of any companies mentioned in the publication should be inferred.

${ }^{a}$ Electronic mail: chanhp@umich.edu

${ }^{1}$ E. L. Gingold, X. Wu, and G. T. Barnes, "Contrast and Dose with MoMo, Mo-Rh, and Rh-Rh target-filter combinations in mammography,', Radiology 195, 639-644 (1995).

${ }^{2}$ A. Maidment, R. Fahrig, and M. J. Yaffe, "Dynamic range requirements in digital mammography,' Med. Phys. 20, 1621-1633 (1993).

${ }^{3}$ P. C. Bunch, K. E. Huff, and R. Van Metter, "Analysis of detective quantum efficiency of radiographic screen/film system,'” J. Opt. Soc. Am. A 4, 902-909 (1987).

${ }^{4}$ A. Stacey-Clear, K. A. McCarthy, D. A. Hall, E. P. Spellman, G. White, C. A. Hulka, G. J. Whitman, E. F. Halpern, and D. B. Kopans, "Mammographically detected breast cancer in women under 50 years old," Radiology 186, 677-680 (1993).

${ }^{5}$ H. R. Blackwell, “Contrast thresholds of the human eye,' J. Opt. Soc. Am. 36, 624-643 (1946)

${ }^{6}$ B. Baxter, H. Ravindra, and R. A. Normann, "Changes in lesion detectability caused by light adaptation in retinal photo-receptors,' Invest. Radiol. 17, 394-401 (1982).

${ }^{7}$ H. L. Snyder, 'Chapter 3: The Visual System: Capabilities and limitations," in Flat-Panel Display and CRTs, edited by L. E. Tannas, Jr. (Van Nostrand Reinhold, New York, 1985).

${ }^{8}$ K. L. Lam and H. P. Chan, "Development of $\mathrm{x}$-ray beam equalization technique in mammography,' Radiology 169(P), 338 (1988).

${ }^{9}$ K. L. Lam and H. P. Chan, "Exposure equalization technique in mammography,' Invest. Radiol. 24, 154-156 (1989).

${ }^{10}$ K. L. Lam and H. P. Chan, "Effects of x-ray beam equalization on mammography imaging,'” Med. Phys. 17, 242-249 (1990).

${ }^{11}$ G. Panayiotakis, H. Likaki, Z. Kolitsi, and J. Dimipoulos, "An anatomical filter for exposure equalization in mammography,' Radiology $\mathbf{1 5}$, 15-17 (1992).

${ }^{12}$ J. M. Sabol, I. C. Soutar, and D. B. Plewes, "Mammographic scanning equalization radiography,', Med. Phys. 20, 1505-1515 (1993).

${ }^{13}$ J. W. Oestmann, B. Stoel, H. Schrijvershof, J. Vrooman, and L. J. Schultze Kool, "Scanning equalization mammography: Preliminary evaluation," Radio Graphics 14, 123-128 (1994).

${ }^{14}$ J. M. Sabol, I. C. Soutar, and D. B. Plewes, "Practical application of a scan-rotate equalization geometry to mammography,' Med. Phys. 23, 1987-1996 (1996).

${ }^{15}$ J. W. Byng, J. P. Critten, and M. J. Yaffe, “Thickness-equalization processing for mammography images,' Radiology 203, 564-568 (1997).

${ }^{16}$ M. M. Goodsitt, H. P. Chan, B. Liu, A. R. Morton, S. V. Guru, S. Keshavmurthy, and N. Petrick, "Classification of compressed breast shape or the design of equalization filters in mammography,' Med. Phys. 25, 
937-948 (1998)

${ }^{17}$ J. M. Boone, I. Duryea, and R. M. Steiner, "Filter wheel equalization for chest radiography: A computer simulation," Med. Phys. 22, 1029-1037 (1995).

${ }^{18}$ A. R. Morton, H. P. Chan, and M. M. Goodsitt, "Automated modelguided breast segmentation algorithm," Med. Phys. 23, 1107-1108 (1996).

${ }^{19}$ D. R. Dance, J. Persliden, and G. A. Carlsson, "Calculation of dose and contrast for two mammography grids," Phys. Med. Biol. 37, 235-245 (1992).
${ }^{20}$ M. J. Berger and J. H. Hubbell, XCOM: Photon cross sections on a personal computer, US Department of Commerce, National Bureau of Standards, Gaithersburg, MD, NBSIR 87-3597 (1987).

${ }^{21}$ H. E. Johns and J. R. Cunningham, The Physics of Radiology, 4th ed. (Thomas, Springfield, IL, 1983).

${ }^{22}$ R. J. Jennings, "Spectral simulation and photon design program suite," FDA, Rockville, MD 20857 (1993).

${ }^{23}$ W. E. Moore, Estimated sensitometric data for Kodak Min-RE film, personal communication, Eastman Kodak Company, Rochester, NY (1995). 